\title{
SISTEMAS DE MANEJO E A MATÉRIA ORGÂNICA DE SOLO DE VÁRZEA COM CULTIVO DE ARROZ(1)
}

\author{
Paulo César do Nascimento ${ }^{(2)}$, Cimélio Bayer ${ }^{(2)}$, Luís de França da \\ Silva Netto ${ }^{(3)}$, Ana Clara Vian ${ }^{(4)}$, Fernando Vieiro ${ }^{(4)}$, Vera Regina \\ Mussoi Macedo ${ }^{(5)} \&$ Élio Marcolin ${ }^{(5)}$
}

\begin{abstract}
RESUMO
Solos de várzea representam um importante depósito de $\mathrm{C}$ atmosférico, cujo efeito de práticas agrícolas na dinâmica da matéria orgânica (MO) necessita ser mais bem entendido. Este estudo foi realizado em experimento de longa duração (11 anos), localizado na Estação Experimental do Arroz de Cachoeirinha (RS), e teve como objetivo avaliar o efeito do sistema plantio direto (SPD) nos estoques de C orgânico, na labilidade da MO e na proteção física da MO em agregados de um Gleissolo Háplico (200 $\mathrm{g} \mathrm{kg}^{-1}$ de argila) cultivado com arroz (Oriza sativa L.) irrigado por inundação, em comparação ao sistema de preparo convencional (SPC). O estoque de $\mathrm{C}$ orgânico da camada de $0-20 \mathrm{~cm}$ não foi afetado pelos sistemas de manejo de solo (38,39 $\mathrm{Mg} \mathrm{ha}^{-1}$ no solo em SPC e 37,36 $\left.\mathrm{Mg} \mathrm{ha}^{-1} \mathrm{em} \mathrm{SPD}\right)$, o que indica que as taxas de decomposição da MO nesse ambiente anaeróbio não foram influenciadas pelo revolvimento do solo. A labilidade da MO nesse solo de várzea, avaliada pela razão C orgânico particulado (COP)/C orgânico associado aos minerais, foi bem superior à labilidade normalmente verificada em solos aerados e, na média da camada de 0-20 cm, não foi alterada pelos sistemas de manejo de solo $(0,24 \mathrm{em}$ SPC e 0,28 em SPD). O aumento da fração leve-oclusa (FLO) foi apenas uma pequena proporção $(9 \%)$ do aumento do teor de C orgânico total no SPD, em comparação ao SPC, na camada superficial $(0-5 \mathrm{~cm})$, indicando que a proteção física da MO no interior de agregados foi pouco expressiva na estabilização da MO neste ambiente de drenagem restrita. Isso se deve, provavelmente, à efêmera agregação e, ou, à facilidade de acesso dos microrganismos e de suas enzimas à decomposição da MO intra-agregados nesse solo devido à saturação por água.
\end{abstract}

Termos de indexação: plantio direto, C orgânico, proteção física.

\footnotetext{
(1) Recebido para publicação em fevereiro de 2008 e aprovado em setembro de 2009.

(2) Professor do Departamento de Solos, Universidade Federal do Rio Grande do Sul - UFRGS. Av. Bento Gonçalves 7712 , CEP 91540-000 Porto Alegre (RS). E-mails: cimelio.bayer@ufrgs.br; 00009911@ufrgs.br

(3) Doutorando em Ciências do Solo, UFRGS. E-mail: luisdefrança@yahoo.com.br

(4) Estudante de Agronomia, UFRGS. Bolsista de Iniciação Científica do CNPq. E-mails: viiann@hotmail.com; fernandovieiro@yahoo.com.br

${ }^{(5)}$ Pesquisador da Estação Experimental do Arroz - IRGA. Av. Bonifácio Carvalho Bernardes 1494, CEP 94930-030 Cachoeirinha (RS). E-mails: vera-macedo@irga.rs.gov.br; elio-marcolin@irga.rs.gov.br
} 


\title{
SUMMARY: SOIL MANAGEMENT EFFECT ON ORGANIC MATTER IN A FLOODED SOIL UNDER RICE IN SOUTHERN BRAZIL
}

\begin{abstract}
Flooded soils are important sinks of atmospheric $C$, hence the effect of agricultural practices on their organic matter (OM) dynamics should be better understood. This study was carried out in an 11 year experiment at IRGA (Instituto Rio Grandense do Arroz) experimental station in Cachoeirinha, Rio Grande do Sul, southern Brazil. The main objectives of this study were to evaluate the effect of no-tillage (NT) on organic C stocks, on OM lability and on the physical protection of OM in soil aggregates of a Gleysol (200 $\mathrm{g} \mathrm{kg}^{-1}$ clay) cultivated with paddy rice (Oryza sativa $L)$ in comparison to conventional tillage $(C T)$. Organic $C(0-20 \mathrm{~cm})$ was not

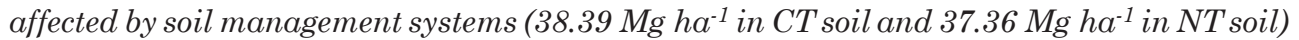
indicating that decomposition rates of OM in this anaerobic environment were not influenced by soil disturbance. The lability of OM in this flooded soil, calculated by the ratio of particulate organic $C(P O C) /$ mineral-associated organic $C$ was higher than the values usually verified for well-drained soils of subtropical regions and, in the 0-20 cm soil layer, $C$ was not affected by soil management systems (0.24 in CT and 0.28 in NT). The increase of the occluded light OM fraction (intra-aggregate light fraction) was only a small proportion (9\%) in relation to the total organic carbon increase in NT, compared to CT, in the surface layer $(0-5 \mathrm{~cm})$. These data suggest that the physical protection of OM in soil aggregates was not an important mechanism of OM stabilization in this environment of restrained drainage. The reason is probably the weak soil aggregation and/or the easy access of microorganisms and their enzymes to the decomposition of the intra-aggregate OM pool due to the soil saturation by water.
\end{abstract}

Index terms: no-tillage, organic carbon, physical protection.

\section{INTRODUÇÃO}

A matéria orgânica (MO) afeta a qualidade física, química e biológica do solo (Mielniczuk et al., 2008) e por esse motivo tem sido utilizada como indicador da qualidade de sistemas de manejo de solo (Vezzani, 2001). Em solos aerados, a adoção de sistemas conservacionistas de manejo, como o sistema plantio direto (SPD), resulta no aumento dos estoques de C orgânico do solo, o qual é mais expressivo nas camadas superficiais do solo e é relacionado ao aporte anual de resíduos vegetais ao solo (Burle et al., 1997; Bayer et al., 2002; Diekow et al., 2005). Em solos de várzea, o aporte de resíduos contribui de maneira similar para o acúmulo de MO (Dobermann \& Witt, 2000; Whitbread et al., 2003; Saharawat, 2005), mas pouco se conhece sobre o efeito de preparos de solo na dinâmica da $\mathrm{MO}$ em solos com drenagem restrita. Nesses solos, a decomposição da MO, na ausência de $\mathrm{O}_{2}$, usualmente ocorre em menores taxas do que em solos aerados e é dependente de receptores de elétrons como íon $\mathrm{Fe}, \mathrm{Mn}$ e sulfato, resultando na oxidação parcial do C orgânico (Neue et al., 1997; Powlson \& Olk, 1999).

A avaliação de frações lábeis da MO em diferentes sistemas de manejo também merece atenção em ambientes com drenagem restrita, pois têm influência marcante na atividade biológica e na química de solos alagados (Gaunt et al., 1995; Saharawat, 2005) e forte ligação com a emissão de metano em solos de várzea; por isso, é grande o interesse sobre a sua dinâmica em solos agrícolas pela sua influência nas emissões desse gás do efeito estufa (Gaunt et al., 1997). Em adição ao estudo do papel de frações lábeis da MO na qualidade do solo e do ambiente, técnicas de fracionamento físico possibilitam identificar mecanismos de estabilização da MO em diferentes solos, e isso pode representar contribuição significativa no entendimento do acúmulo de MO em solos sob sistemas de manejo conservacionista (Jastrow \& Miller, 1998; Conceição et al., 2008).

A estabilização da MO pela interação com a fração mineral do solo, seja pela menor acessibilidade dos microrganismos e de suas enzimas a compostos orgânicos localizados no interior de agregados (proteção física), seja pela formação de complexos altamente estáveis entre grupos funcionais orgânicos e grupos funcionais presentes na superfície dos minerais (estabilidade química), tem sido considerada o principal fator envolvido na preservação dos estoques de C orgânico em solos agrícolas (Zotarelli et al., 2007). Entretanto, estudos dessa natureza têm sido restritos a solos aerados, com a hipótese de que a agregação efêmera e a saturação do solo com drenagem restrita de solos de várzea determinam que a proteção física da MO é um mecanismo pouco eficiente na sua estabilização e, consequentemente, resulta num acúmulo pouco expressivo de C orgânico em solos de várzea submetidos ao SPD.

Este estudo teve como objetivo avaliar o efeito da utilização, por 11 anos, do SPD sobre os estoques de C orgânico, na labilidade da MO e na proteção física da MO em agregados de um Gleissolo Háplico cultivado com arroz irrigado no sul do Brasil, em comparação ao SPC. 


\section{MATERIAL E MÉTODOS}

Amostras de solo foram coletadas em experimento de sistemas de manejo de solo, com 11 anos de duração, localizado no Centro Experimental do Instituto Rio Grandense do Arroz (IRGA), no município de Cachoeirinha (RS), cujas coordenadas geográficas são $29^{\circ} 56$ ' S e $50^{\circ} 08$ ' W. O clima na região corresponde ao tipo Cfa (subtropical de verão úmido quente), segundo classificação de Köppen. O solo apresenta, em média, na camada de $0-20 \mathrm{~cm}, 200 \mathrm{~g} \mathrm{~kg}^{-1}$ argila e $350 \mathrm{~g} \mathrm{~kg}^{-1}$ de areia, tendo a caulinita como mineral predominante na fração argila (Costa, 2005).

O experimento consistiu em dois sistemas de manejo de solo (preparo convencional - SPC, plantio direto - SPD), de acordo com um delineamento de blocos casualizados, em três repetições. Quando da sua implantação, foi realizada sistematização da área em quadros, e, a cada ano, foram realizadas apenas as operações referentes aos manejos de solo sem nova sistematização. A cada ano foi cultivado arroz (Oryza sativa $\mathrm{L}$.) no verão e, no inverno, houve estabelecimento de azevém (Lolium multiflorum L.) por ressemeadura natural. O SPC consistiu em preparo do solo por aração, seguida de duas gradagens com grade leve em setembro-outubro de cada ano, visando à adequação da área para semeadura. O SPD consistiu no uso de controle químico do azevém e semeadura sobre os restos culturais dessa espécie. Uma área sob vegetação natural (VN), com predomínio de gramíneas, a qual permaneceu durante longo período (cerca de 15 anos) em pousio, foi amostrada como referência aproximada da condição natural do solo. Mais detalhes dos tratamentos e da condução experimental são descritos em Costa (2005).

A coleta das amostras de solo foi realizada em trincheiras, em outubro de 2005, anterior às operações de manejo de solo, nas profundidades de $0-5,5-10$ e 10-20 cm. O solo foi seco ao ar. Uma subamostra de aproximadamente $100 \mathrm{~g}$ foi moída em gral de ágata até passar em peneira de $0,15 \mathrm{~mm}$ e analisada em relação aos teores de $\mathrm{C}$ orgânico por combustão seca em analisador Shimadzu TOC-VCSH. Na porção restante da amostra de solo não moída, os agregados naturais foram passados em peneira de $9 \mathrm{~mm}$ e submetidos a fracionamentos granulométrico e densimétrico. No fracionamento granulométrico, a fração particulada $(>53 \mu \mathrm{m})$ foi obtida a partir da agitação da suspensão de $20 \mathrm{~g}$ de solo com $80 \mathrm{~mL}$ de hexametafosfato de sódio $\left(5 \mathrm{~g} \mathrm{~L}^{-1}\right)$ em agitador horizontal, por $16 \mathrm{~h}$, e passagem em peneira com auxílio de jato de água. O material retido na peneira, cuja fração é denominada $\mathrm{C}$ orgânico particulado (COP), foi seco, quantificado em relação à sua massa, moído a $0,15 \mathrm{~mm}$ e analisado quanto ao teor de $\mathrm{C}$ em analisador Shimadzu - VCSH (Cambardella \& Elliot, 1992). O estoque de $\mathrm{C}$ associado aos minerais foi calculado por diferença entre os estoques de $\mathrm{C}$ orgânico total e de C orgânico particulado (Bayer et al., 2002).
O fracionamento densimétrico foi realizado apenas na camada de $0-5 \mathrm{~cm}$, conforme procedimento descrito em Conceição et al. (2008). O fracionamento consistiu em agitação manual branda do tubo de centrífuga contendo 10 g de solo e $80 \mathrm{~mL}$ de politungstato de sódio $2 \mathrm{~g} \mathrm{~cm}^{-3}$. Após deixar $1 \mathrm{~h}$ para decantação do material e centrifugação por $60 \mathrm{~min}$ a $2.000 \mathrm{~g}$, o sobrenadante foi removido e passado em filtro de fibra de vidro previamente quantificado em relação à sua massa. Esta fração orgânica, a qual consiste na fração levelivre (FLL), foi seca, pesada e moída em gral de ágata. O material no fundo do tubo de centrífuga foi submetido à dispersão por sonificação a $450 \mathrm{~J} \mathrm{~mL}^{-1}$, suficiente para dispersão total do solo, conforme verificado em ensaio com aplicação de níveis de energia de ultrassom crescentes (dados não apresentados).

O sobrenadante contendo a fração leve-oclusa (FLO) foi centrifugado e passou pelos mesmos procedimentos realizados para obtenção da FLL, sendo ambas analisadas quanto aos teores de $\mathrm{C}$ em analisador Shimadzu. O estoque de C na fração pesada (FP) foi calculado por diferença entre os estoques de $\mathrm{C}$ orgânico total e de C na fração leve total da MO (FLL + FLO). $\mathrm{O}$ solo, em todos os tratamentos, foi analisado quanto à sua densidade (Quadro 1), e os valores utilizados para o cálculo de estoques de C no solo inteiro e em todas as frações da MO em massas equivalentes de solo (Ellert \& Bettany, 1995; Bayer, 2003), utilizando o solo sob vegetação natural como referência.

As taxas de decomposição anual da MO, nos dois sistemas de manejo, foram calculadas segundo procedimento descrito em Bayer et al. (2006). Para isso, considerou-se que os estoques de $\mathrm{C}$ orgânico no solo estavam em equilíbrio ( $\mathrm{dC} / \mathrm{dt}=$ zero), condição na qual há igualdade entre as perdas e adições de $\mathrm{C}$ orgânico $\left(\mathrm{k}_{1} \mathrm{~A}=\mathrm{k}_{2} \mathrm{C}\right)$ no solo. Além disso, atribuíramse adições de massa seca (A) de $2 \mathrm{Mg}$ ha $^{-1}$ pelas culturas de inverno (invasoras e azevém) e de $6 \mathrm{Mg} \mathrm{ha}^{-1}$ pelo arroz no verão (índice de colheita de 0,5 ), uma contribuição de raízes equivalente a $30 \%$ da adição da parte aérea das culturas e um coeficiente de humificação $\left(\mathrm{k}_{1}\right)$ de 0,14 (Bayer et al., 2006). As taxas de decomposição anual da MO no solo em SPC e SPD foram calculadas de acordo com a expressão $\mathrm{k}_{2}=\mathrm{k}_{1} \cdot \mathrm{A} /$ Csolo.

Quadro 1. Valores de densidade de um Gleissolo Háplico sob diferentes sistemas de uso e manejo (VN-vegetação natural, SPC-sistema preparo convencional e SPD-sistema plantio direto)

\begin{tabular}{cccc}
\hline \multirow{2}{*}{ Preparo de solo } & \multicolumn{3}{c}{ Profundidade (cm) } \\
\cline { 2 - 4 } & $\mathbf{0 - 5}$ & $\mathbf{5}-\mathbf{1 0}$ & $\mathbf{1 0 - 2 0}$ \\
\cline { 2 - 4 } & & & \\
& & & \\
& & & \\
VN dm & -3 & \\
SPC & 1,53 & 1,81 & 1,79 \\
SPD & 1,44 & 1,67 & 1,62 \\
& 1,32 & 1,81 & 1,77 \\
\hline
\end{tabular}


Os resultados foram submetidos à análise da variância, e a diferença entre médias dos sistemas de manejo foi avaliada pelo teste de Tukey a $5 \%$, separadamente para cada camada de solo. O solo sob vegetação natural não foi submetido à análise estatística, pois não segue o delineamento experimental dos demais tratamentos.

\section{RESULTADOS E DISCUSSÃO}

\section{Estoques de C orgânico}

O estoque de C orgânico no perfil do solo em SPD foi similar ao do solo sob VN, os quais apresentaram maiores estoques em relação ao SPC (cerca de $56 \%$ ) na camada de $0-5 \mathrm{~cm}$, decorrente da deposição de resíduos vegetais sobre o solo (Quadro 2). Por sua vez, o solo em SPC apresentou uma distribuição mais uniforme dos teores de $\mathrm{C}$ orgânico na camada de 0 $20 \mathrm{~cm}$, decorrente da mistura dos resíduos vegetais nessa camada "arável" do solo. Os maiores estoques de C na camada superficial do solo em SPD foram contrabalanceados por maiores estoques de $\mathrm{C}$ na camada de 10-20 cm do solo em SPC (Quadro 2). Devido a esses acúmulos compensatórios de $\mathrm{C}$ nas diferentes camadas de solo, não se verificou diferença nos estoques de $\mathrm{C}$ orgânico no solo, na camada de 0-20 cm, após 11 anos de utilização dos diferentes sistemas de manejo de solo (38,39 $\mathrm{Mg} \mathrm{ha}^{-1}$ em SPC e 37,36 $\mathrm{Mg} \mathrm{ha}^{-1}$ em SPD).
A taxa de decomposição da MO estimada no solo foi de aproximadamente 1,5\%, a qual não foi afetada pelos sistemas de manejo de solo. Essa taxa de decomposição da MO é inferior às taxas que têm sido estimadas para Argissolo aerado com textura (220 $\mathrm{g} \mathrm{kg}^{-1}$ argila) e mineralogia (predomínio de caulinita) similares, sob as mesmas condições climáticas, cuja taxa de decomposição da MO foi estimada em 1,9 \% em SPD e em $4 \%$ em SPC (Bayer et al., 2006). Essas diferenças indicam que, em caso de alagamento do solo, a taxa de decomposição da $\mathrm{MO}$ é reduzida devido ao efeito da anaerobiose na atividade microbiana nesses solos (Oades, 1988; Polwson \& Olk, 2000; Whitbread et al., 2003) e que, principalmente, os fatores determinantes da estabilização da MO em solos aerados sob SPD possivelmente não se manifestam de forma tão intensa em solos de várzea.

\section{Estoques de C orgânico particulado e labilidade da matéria orgânica}

$\mathrm{O}$ estoque de $\mathrm{C}$ orgânico na fração particulada (> $53 \mu \mathrm{m}$ ) representou, na camada de 0-5 cm, aproximadamente $30 \%$ do estoque de C orgânico do solo (Quadro 2). Em solos aerados sob clima subtropical como os deste estudo, essa fração lábil da MO representa, normalmente, de 10 a $20 \%$ do estoque de C orgânico (Bayer et al., 2002; Bayer, 2003; Vieira et al., 2007). A maior proporção do C orgânico particulado nesse solo de várzea, comparado a solos aerados, devese provavelmente ao efeito de excesso hídrico, o que limita a atividade microbiana do solo e contribui para

Quadro 2. Estoques de C orgânico total, C orgânico particulado e C orgânico associado aos minerais em Gleissolo Háplico sob vegetação natural (VN), sistema de preparo convencional (SPC) e sistema plantio direto (SPD)

\begin{tabular}{|c|c|c|c|c|}
\hline \multirow{2}{*}{ Preparo de solo } & \multicolumn{4}{|c|}{ Profundidade $(\mathrm{cm})$} \\
\hline & $0-5$ & $5-10$ & $10-20$ & 0-20 \\
\hline \multicolumn{5}{|c|}{$\mathrm{C}$ orgânico total, $\mathrm{Mg} \mathrm{ha}^{-1}$} \\
\hline $\mathrm{VN}$ & $16,32( \pm 1,88)$ & $7,68( \pm 0,40)$ & $11,92( \pm 0,26)$ & $35,92( \pm 4,47)$ \\
\hline $\mathrm{SPC}$ & $10,45 \mathrm{~b}$ & $11,09 \mathrm{a}$ & $16,95 \mathrm{a}$ & $38,39 \mathrm{a}$ \\
\hline SPD & $16,38 \mathrm{a}$ & $9,18 \mathrm{a}$ & $11,80 \mathrm{~b}$ & $37,36 \mathrm{a}$ \\
\hline \multicolumn{5}{|c|}{$\mathrm{C}$ orgânico particulado, $\mathrm{Mg} \mathrm{ha}^{-1}$} \\
\hline $\mathrm{VN}$ & $5,10( \pm 1,01)$ & $1,36( \pm 0,18)$ & $1,87( \pm 0,42)$ & $8,34( \pm 1,37)$ \\
\hline SPC & $3,12 \mathrm{~b}$ & $2,44 \mathrm{a}$ & $1,80 \mathrm{a}$ & $7,36 \mathrm{a}$ \\
\hline SPD & $5,86 \mathrm{a}$ & $1,04 \mathrm{~b}$ & $1,37 \mathrm{a}$ & $8,28 \mathrm{a}$ \\
\hline \multicolumn{5}{|c|}{$\mathrm{C}$ orgânico associado aos minerais, $\mathrm{Mg}_{\mathrm{ha}}{ }^{-1}$} \\
\hline $\mathrm{VN}$ & $11,20( \pm 1,79)$ & $6,32( \pm 0,34)$ & $10,05( \pm 0,33)$ & $27,58 \quad( \pm 3,37)$ \\
\hline $\mathrm{SPC}$ & $7,32 \mathrm{~b}$ & 8,66 a & $15,05 \mathrm{a}$ & 31,03 a \\
\hline SPD & $10,52 \mathrm{a}$ & $8,11 \mathrm{a}$ & $10,43 \mathrm{~b}$ & $29,08 \mathrm{a}$ \\
\hline
\end{tabular}

Letras diferentes na coluna, para cada variável, indicam diferença entre as médias dos sistemas de manejo de solo pelo teste de Tukey a $5 \%$; resultados da vegetação natural não foram submetidos à análise de variância; os valores entre parênteses correspondem ao desvio-padrão. 
a manutenção de maiores estoques de $\mathrm{C}$ em frações lábeis da MO no solo (Gaunt et al., 1995; Olk et al., 2000b).

No solo em SPD, o estoque de C orgânico particulado foi de $5,86 \mathrm{Mg} \mathrm{ha}^{-1}$ na camada de $0-5 \mathrm{~cm}$, quase o dobro do estoque verificado no solo em SPC (3,12 $\left.\mathrm{Mg} \mathrm{ha}^{-1}\right)$. Esse acúmulo de $88 \%$ do estoque de MO lábil na camada superficial do solo em SPD devese, em grande parte, à adição proporcionalmente maior de resíduos vegetais na superfície do solo, e no SPC os resíduos vegetais são incorporados na camada de 0 $20 \mathrm{~cm}$. Por outro lado, essa incorporação dos resíduos no SPC determinou um acúmulo de $\mathrm{C}$ na camada subsuperficial $(5-10 \mathrm{~cm})$. Considerando a camada de 0-20 cm, não se verificou efeito significativo dos sistemas de manejo de solo no estoque de $\mathrm{C}$ orgânico particulado, à semelhança do que foi verificado nos estoques de $\mathrm{C}$ orgânico total.

O C orgânico associado aos minerais representou aproximadamente 70 a $80 \%$ do C orgânico total, e isso representa a fração mais humificada da MO do solo (Quadro 2). Apesar de apresentar tempo de ciclagem mais longo no solo, o que determina que um período mais longo de tempo seja necessário para que o efeito de práticas de manejo seja observado nos estoques de $\mathrm{C}$ orgânico nessa fração, verificou-se uma tendência de aumento no estoque de $\mathrm{C}$ nessa fração orgânica na camada superficial $(0-5 \mathrm{~cm})$ do solo em SPD (10,52 Mg ha ${ }^{-1}$ de C), em comparação ao solo em $\operatorname{SPC}\left(7,32 \mathrm{Mg} \mathrm{ha}^{-1}\right.$ de C). Por sua vez, a incorporação de $\mathrm{C}$ proveniente dos resíduos culturais da lavração e gradagem também favoreceu o acúmulo do $\mathrm{C}$ associado aos minerais no solo em SPC (15,05 $\mathrm{Mg} \mathrm{ha}^{-1}$ de C), em comparação ao SPD $\left(10,43 \mathrm{Mg} \mathrm{ha}^{-1}\right.$ de C) na camada de 10-20 cm (Quadro 2).

A razão C orgânico particulado/C orgânico associado aos minerais é um índice de labilidade da MO do solo (Dieckow et al., 2005; Vieira et al., 2007). Verificou-se que o solo em SPD apresentou maior labilidade do C na camada superficial, enquanto o solo em SPC apresentou maior labilidade na camada de 5-10 cm (Figura 1). Essa diferenciação na labilidade da MO entre os sistemas é decorrente das diferentes camadas nas quais são adicionados os resíduos culturais de arroz no verão e do azevém e invasoras no inverno. Se, por um lado, a maior labilidade da MO na camada superficial do solo pode ser um aspecto favorável à maior atividade microbiana com reflexos nas condições físicas e químicas do solo, por outro, pode resultar em maiores emissões de metano nesses solos com drenagem restrita (Gaunt et al., 1997; Neue et al., 1997). Essa pode ser uma explicação para as maiores emissões de metano verificadas no solo em SPC nesse mesmo experimento, em comparação ao SPD (Costa, 2005). O maior teor de C lábil numa camada $(5-10 \mathrm{~cm})$ mais reduzida do que na camada superficial do solo $(0-5 \mathrm{~cm})$ determina uma condição potencial de maior emissão de metano para atmosfera no solo em SPC do que no SPD.

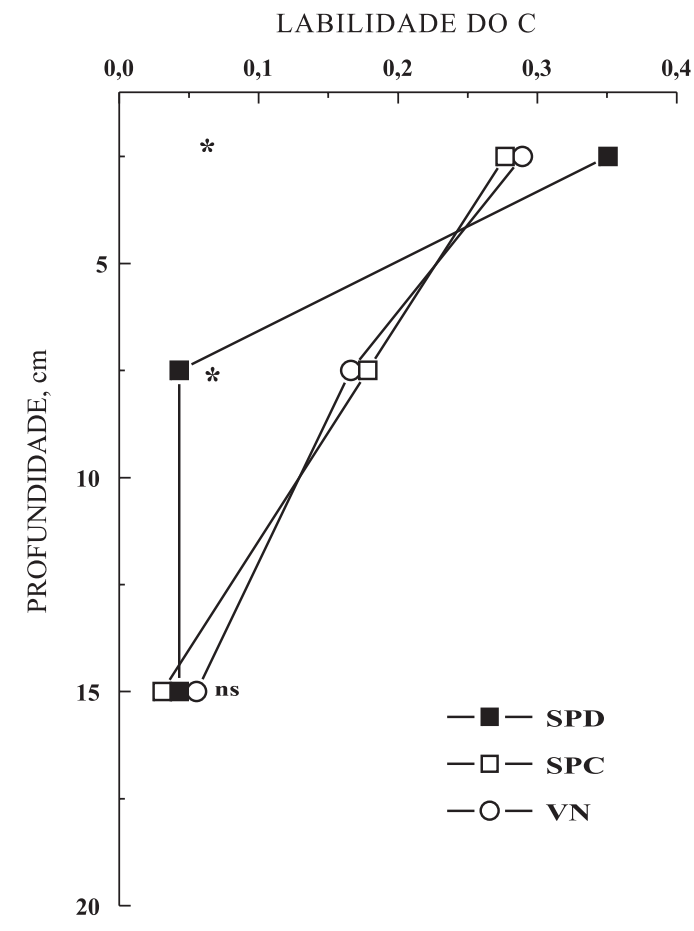

Figura 1. Labilidade do carbono orgânico (razão C particulado/C associado aos minerais) em Gleissolo Háplico sob vegetação natural (VN), sistema de preparo convencional (SPC) e sistema plantio direto (SPD). *: médias dos tratamentos SPC e SPD, em cada profundidade de solo, diferem entre si pelo teste de Tukey a $5 \%$; ns: diferença não significativa; resultados da VN não foram submetidos à análise de variância.

De maneira geral, as diferenças verificadas na labilidade da MO entre os sistemas de preparo de solo foram menos expressivas do que tem sido normalmente verificado em solos aerados (Dieckow et al., 2005; Vieira et al., 2007). Isso se deve ao fato de que os sistemas de manejo de solo praticamente não se diferenciaram quanto ao seu efeito na taxa de decomposição da MO do solo, e essa similaridade nas taxas de decomposição determinou semelhantes estoques de $\mathrm{C}$ orgânico e labilidade da MO nesse solo de várzea.

\section{Mecanismos de estabilização da MO no solo}

Os teores de C na fração leve-livre (FLL), fração leve-oclusa (FLO) e fração pesada (FP), avaliados na camada de $0-5 \mathrm{~cm}$, obedeceram à seguinte ordem: FP $>$ FLO $>$ FLL. Na média dos preparos de solo, os teores de C nessas frações foram de 1,45, 3,05 e $13,15 \mathrm{~g} \mathrm{~kg}^{-1} \mathrm{de} \mathrm{C}$ solo, representando uma contribuição de 8,18 e $74 \%$ do estoque de C orgânico total, respectivamente. Esses diferentes teores e proporções de $\mathrm{C}$ se devem à diferente estabilidade do $\mathrm{C}$ nas frações físicas da $\mathrm{MO}$ no solo. Na FLL, apenas a recalcitrância química contribuiu para a estabilidade da matéria 

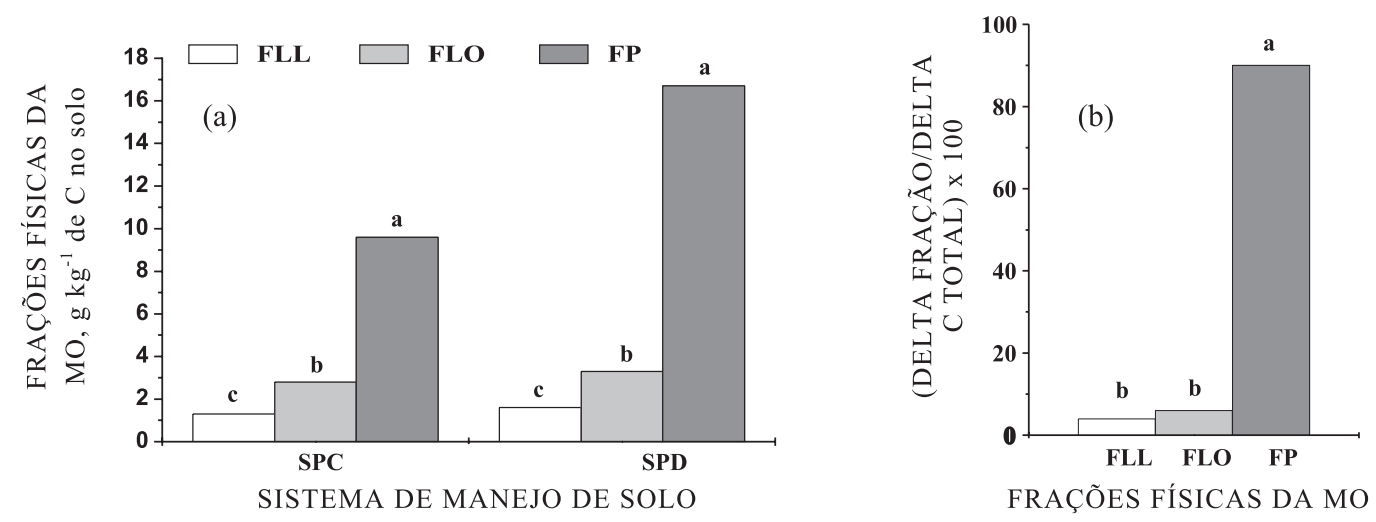

Figura 2. Estoques de C nas frações leve-livre (FLL), leve-oclusa (FLO) e pesada (FP) da matéria orgânica (MO) no solo em sistema preparo convencional (SPC) e plantio direto (SPD) (a) e proporção do C acumulado em cada fração física em relação ao total de C acumulado sob SPD em comparação ao SPC (b) na camada de 0-5 cm de um Gleissolo Háplico cultivado com arroz irrigado. Letras diferentes, dentro de cada sistema de preparo de solo, indicam diferença entre as frações físicas da MO pelo teste de Tukey a 5 \% (a), e letras diferentes indicam diferença na proporção de $\mathrm{C}$ acumulado nas frações físicas da MO do solo no sistema SPD em comparação ao solo no sistema SPC (b).

orgânica, enquanto na FLO a proteção física da matéria orgânica teve efeito aditivo à recalcitrância química na estabilização da matéria orgânica. Por sua vez, os maiores teores de $\mathrm{C}$ na FP devem-se à ocorrência da recalcitrância química, à proteção física e à estabilidade coloidal pela interação da MO com a fração mineral do solo (Jastrow \& Miller, 1998).

Com base na relação entre as frações físicas da MO e os mecanismos de estabilização atuantes, os teores (2,90 e 2,23 $\mathrm{g} \mathrm{kg}^{-1}$ de $\mathrm{C}$ no solo) e proporções (17 e $18 \%$ do C orgânico total) similares entre C- FLL e C-FLO sugerem baixa magnitude da proteção física na estabilização da MO nesse solo de várzea. Esses resultados são bastante distintos daqueles que normalmente são obtidos em solos aerados, nos quais o estoque de $\mathrm{C}$ na FLO contém aproximadamente o dobro do estoque de $\mathrm{C}$ daquele verificado na FLL (Conceição et al., 2008).

O SPD promoveu o acúmulo de $\mathrm{C}$ principalmente na FP (84\%) da MO, enquanto o acúmulo na FLO respondeu pelo acúmulo de apenas $9 \%$ de $\mathrm{C}$ em comparação ao solo em SPC (Figura 2). Esses resultados reforçam a baixa magnitude da proteção física da MO nesse solo mal drenado e contrastam com resultados obtidos em solos aerados, os quais evidenciam que quantidades equivalentes ou superiores a $30 \%$ do acúmulo de $\mathrm{C}$ verificadas em solos em SPD ocorrem na FLO (Tan et al., 2007; Zotarelli et al., 2007; Conceição et al., 2008), indicando que a proteção da MO no interior de agregados é menos expressiva em solos hidromórficos que em solos aerados. O excesso de água pode ser responsável pelo efeito pouco expressivo dos agregados na diminuição do acesso das enzimas e microrganismos à decomposição da MO. Por sua vez, o acúmulo preferencial de $\mathrm{C}$ na $\mathrm{FP}$ neste solo com drenagem restrita em SPD pode estar relacionado ao intenso processo de interação propiciado nesse ambiente entre a MO e os minerais da fração argila (Olk \& Senesi, 2000).

\section{CONCLUSÕES}

1. O sistema plantio direto promove acúmulo de $\mathrm{C}$ orgânico na camada superficial de solos hidromórficos, o que é contrabalanceado pelo acúmulo de $\mathrm{C}$ nas camadas subsuperficiais dos solos em preparo convencional.

2. A matéria orgânica do solo apresentou alta labilidade, evidenciando o efeito do ambiente anaeróbio na determinação de baixa taxa de decomposição da matéria orgânica no solo.

3. A proteção física da matéria orgânica no interior de agregados foi pouco expressiva neste solo com drenagem restrita em plantio direto, o que pode estar relacionado ao efeito do excesso de água no processo de agregação e à facilidade de acesso de microrganismos intra-agregados e suas enzimas à matéria orgânica.

\section{AGRADECIMENTOS}

Ao Conselho Nacional de Desenvolvimento Científico e Tecnológico $(\mathrm{CNPq})$ pelo auxílio financeiro no âmbito do projeto 474919/2007-2 (Edital Universal 2007. Cimélio Bayer agradece ao CNPq pela bolsa de produtividade em pesquisa. 


\section{LITERATURA CITADA}

BAYER, C. Aumento do estoque de matéria orgânica de um Cambissolo Húmico catarinense sob plantio direto. R. Ci. Agrovet., 2:81-87, 2003.

BAYER, C.; MIELNICZUK, J.; MARTIN-NETO, L. \& ERNANI, P.R. Stocks and humification degree of organic matter soil as affected by no-tillage on a subtropical soil. Plant Soil, 238:133-140, 2002.

BAYER, C.; LOVATO, T.; DIECKOW, J.; ZANATTA, J.A. \& MIELNICZUK, J. A method for estimating coefficients of soil organic matter dynamics based on long-term experiments. Soil Till. Res., 91:217-226, 2006.

BURLE, M.L.; MIELNICZUK, J. \& FOCCHI, S. Effect of cropping systems on soil chemical characteristics, with emphasis on soil acidification. Plant Soil, 190:309-316, 1997.

CAMBARDELLA, C.A. \& ELLIOT, E.T. Particulate soil organic matter changes across a grassland cultivated sequence. Soil Sci. Soc. Am. J., 56:777-783, 1992.

CONCEIÇÃO, P.C.; BOENI, M.; DIECKOW, J.; BAYER, C. \& MIELNICZUK, J. Fracionamento densimétrico com politungstato de sódio no estudo da proteção física da matéria orgânica em solos. R. Bras. Ci. Solo, 32:541-549, 2008.

COSTA, F.S. Estoques de carbono orgânico e efluxo de dióxido de carbono e de metano de solos em preparo convencional e plantio direto no subtrópico brasileiro. Porto Alegre, Universidade Federal do Rio Grande do Sul, 2005.128p. (Tese de Doutorado)

DIECKOW, J.; MIELNICZUK, J.; KNICKER, H.; BAYER, C.; DICK, D.P.\& KOGEL-KNABNER, I. Carbon and nitrogen stocks in physical fractions of a subtropical Acrisol as influenced by long-term no-till cropping system and $\mathrm{N}$ fertilization. Plant Soil, 268:319-328, 2005.

DOBERMANN, A. \& WITT, C. The potential impact of crop intensification on carbon and nitrogen cycling in intensive rice systems. In: KIRK, G.J.D. \& OLK, D.C., eds. Carbon and nitrogen dynamics in flooded soils. Los Baños, International Rice Research Institute, 2000. p.1-26.

ELLERT, B.H. \& BETTANY, J.R. Calculation of organic matter and nutrients stored in soils under contrasting management regimes. Can. J. Soil Sci., 75:529-538, 1995.

GAUNT, J.L.; NEUE, H.U. \& BRAGAIS, J. Soil characteristics that regulate soil reduction and methane production in wetland rice soils. Soil Sci. Soc. Am. J., 61:1526-1531, 1997.

GAUNT, J.L.; NEUE, H.U. \& CASSMAN, K.G. Microbial biomass and organic matter turnorver in wetland rice soils. Biol. Fert. Soils, 19:333-342, 1995.
JASTROW, J.D. \& MILLER, R.M. Soil aggregate stabilization and carbon sequestration: Feedbacks through organomineral associations. In: LAL, R.; KIMBLE, J.M.; FOLLET, R.F. \& STEWART, B.A., eds. Soil processes and the carbon cycle, 1998. p.207-224.

MIELNICZUK, J. Matéria orgânica e a sustentabilidade dos sistemas agrícolas. In: SANTOS, G.A.; SILVA, L.S.; CANELLAS, L.P. \& CAMARGO, F.A.O., eds. Fundamentos da matéria orgânica do solo - ecossistemas tropicais e subtropicais. 2.ed. Porto Alegre, Metrópole, 2008. p.1-5.

OLK, D. \& SENESI, R. Properties of chemically extracted soil organic matter in intensively cropped lowland rice soils. In: KIRK, G.J.D. \& OLK, D.C., eds. Carbon and nitrogen dynamics in flooded soils. Los Baños, International Rice Research Institute, 2000a. p.65-87.

OLK, D.; KESSEL, C.van \& BRONSON, K.F. Managing soil organic matter in rice and nonrice soils: Agronomic questions. In: KIRK, G.J.D. \& OLK, D.C., eds. Carbon and nitrogen dynamics in flooded soils. Los Baños, International Rice Research Institute, 2000b. p.27-47.

NEUE, H.U.; GAUNT, J. L.; WANG, Z.P.; BECKERHEIDMANN, P. \& QUIJANO, C. Carbon in tropical wetlands. Geoderma, 79:163-185, 1997.

OADES, J.M. The retention of organic matter in soils. Biogeochemistry, 5:35-70, 1988.

POWLSON, D.S. \& OLK, D.C. Long-term soil organic matter dynamics. In: KIRK, G.J.D. \& OLK, D.C., eds. Carbon and nitrogen dynamics in flooded soils. Los Baños, International Rice Research Institute, 2000. p.49-63.

SAHARAWAT, K.L. Fertility and organic matter in submerged rice soils. Curr. Sci., 88:735-739, 2005.

TAN, Z.; LAL, R.; OWENS, L. \& IZAURRALDE, R.C. Distribution of light and heavy fractions of soil organic carbon as related to land use and tillage pratice. Soil Till. Res., 93:53-59, 2007.

WHITBREAD, A.; BLAIR, G.; KONBOON, Y.; LEFROY, R. \& NAKLANG, K. Managing crop residues, fertilizers and leaf litters to improve soil $\mathrm{C}$, nutrient balances, and the grain yield of rice and wheat cropping systems in Thailand and Australia. Agric. Ecosyst. Environ., 100:251-263, 2003.

VEZZANI, F. M. Qualidade do sistema solo na produção agrícola. Porto Alegre, Universidade Federal do Rio Grande do Sul, 2001. 184p. (Tese de Doutorado)

VIEIRA, F.C.B.; BAYER, C.; ZANATTA, J.A.; DIECKOW, J. \& MIELNICZUK, J. Carbon management index based on physical fractionation of soil organic matter in an Acrisol under long-term no-till cropping systems. Soil Till. Res., 96:195-204, 2007

ZOTARELLI, L.; ALVES, B.J.R.; URQUIAGA, S.; BODDEY, R.M. \& SIX, J. Impact of tillage and crop rotation on light fraction and intra-agregate soil organic matter in two oxisols. Soil Till. Res., 95:196-206, 2007. 